Special issue of the 3rd International Conference on Computational and Experimental Science and Engineering (ICCESEN 2016)

\title{
Determination of the Fatigue Behavior of a Wheel Rim Using Finite Element Analysis
}

\author{
E. ESENER ${ }^{a}$, S. $\operatorname{ERCAN}^{b}$ AND M. FiRAT ${ }^{b, *}$ \\ ${ }^{a}$ Bilecik Seyh Edebali University, Mechanical Engineering Department, Bilecik, Turkey \\ ${ }^{b}$ Sakarya University, Mechanical Engineering Department, Sakarya, Turkey
}

\begin{abstract}
Automobile wheel rims are critical elements that work under fatigue loading. For this reason, it is very important to determine fatigue damage of the wheel rims. Today, finite element analysis is used to make accurate predictions. In this study, it is aimed to determine Chaboche damage model parameters for an aluminium alloy wheel rim. Msc Marc software is used for finite element analysis. First, Chaboche damage model parameters are obtained using simple Holloman equation for finite element analysis. Model parameters are validated by single element tests. Then, fatigue behaviour of an automobile wheel rim is analysed with verified Chaboche parameters. At last, critical areas under fatigue loading are identified on the wheel rim.
\end{abstract}

DOI: 10.12693/APhysPolA.132.617

PACS/topics: 87.10.Kn, 81.70.Bt

\section{Introduction}

Wheels are critical components in automotive industry, which are exposed to variable stresses under static and dynamic forces. Many tests are determined by automotive and wheel manufacturers to perform on designed wheel models [1-5].

This study aims to determine Chaboche material model parameters, which in turn will be utilized to examine fatigue behaviour of an aluminium alloy wheel. Predefined material is verified via single element test and thereafter a finite element model is prepared to simulate the fatigue strength by wheel cornering test. Chaboche material model is used to predict damage in wheel cornering process [6-8]. Chaboche nonlinear kinematic hardening model has eliminated the disadvantages of ArmstrongFrederick model, by creating a backstress through superposition of M parts.

$$
X=\frac{C}{\gamma}\left(1-\mathrm{e}^{-\gamma \varepsilon_{\mathrm{pl}}}\right) .
$$

\section{Materials and methods}

In accordance with Holloman equation [9], cyclic plasticity behaviour of the aluminium alloy is modelled with a finite element method, using Chaboche material model. Thus material properties are determined. Since Chaboche material model is used in fatigue strength calculations, the small strain locations are specifically examined with additional sensitivity. Material yield strength is assumed to be $30 \mathrm{MPa}$ in this study.

In commercial Mentat software, to use the Chaboche material model, constants $C$ and $\gamma$ need to be determined, to obtain the strain accumulation graph. For this

* corresponding author; e-mail: firat@sakarya.edu.tr

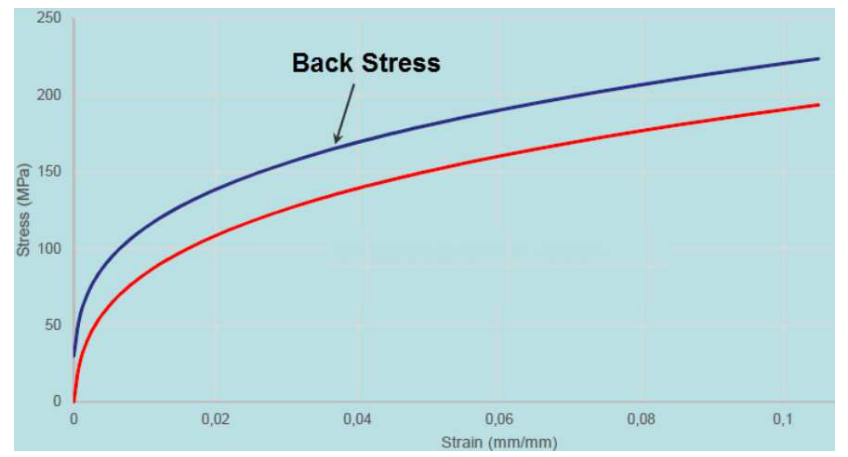

Fig. 1. Stress-strain and back stress-strain curves of employed material.

TABLE I

Chaboche parameters of proposed model.

\begin{tabular}{c|c|c}
\hline \hline$\varepsilon$ & $C$ & $\gamma$ \\
\hline 0 & 34100 & 539 \\
0.004 & 34100 & 539 \\
0.00425 & 20980 & 229.2 \\
0.01 & 20980 & 229.2 \\
0.011 & 11620 & 87.11 \\
0.03 & 11620 & 87.11 \\
0.031 & 7575 & 45.37 \\
0.05 & 7575 & 45.37 \\
0.055 & 4994 & 23.89 \\
0.105 & 4994 & 23.89
\end{tabular}

purpose Matlab commercial software is used for curve fitting purposes. Marc commercial software can only utilise single term Chaboche material model, as a consequence, the curve fitting procedure is conducted by dividing the stress-strain curve into certain intervals. Stress-strain and back stress-strain curves are shown in Fig. 1 and Chaboche parameters for aluminium alloy can be seen in Table I. 


\subsection{Single element test}

To verify obtained values a single element test for a eight-node cubic element was conducted. The model was prepared using $\mathrm{mm}$ as a unit. After entering node coordinates these nodes are selected to build the element (Fig. 2). Nodes 3, 4, 7 and 8 have $x$-axis symmetry while nodes $1,2,3$ and 4 have $y$-axis symmetry and nodes $2,3,6$ and 7 have $z$-axis symmetry. Loading is applied in $y$ direction with a total value of $0.1 \mathrm{~mm}$. Loading is distributed equally between nodes $5,6,7$ and 8 . Single element test is conducted for Chaboche material model for a total of five cycles (Fig. 3). As a result, it has been observed that the curve obtained via finite element modeling is similar to Holloman equation curve.

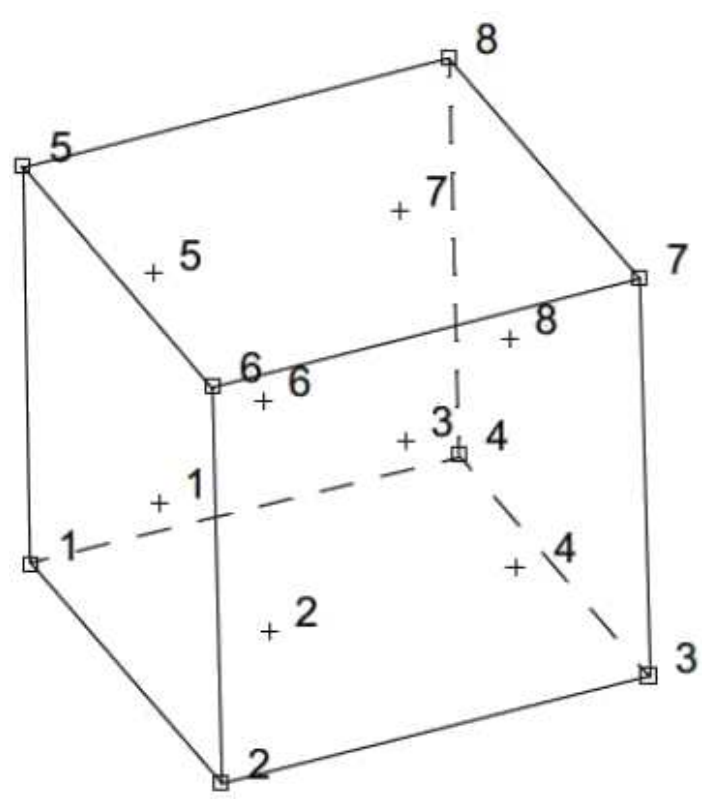

Fig. 2. Construction of single element for verification.

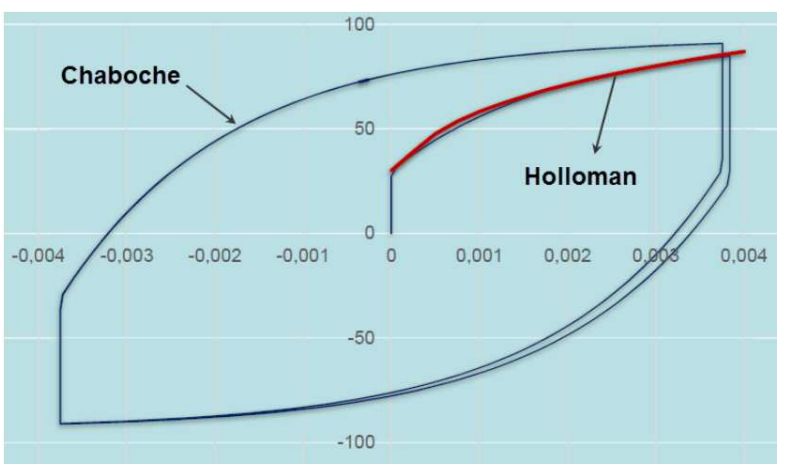

Fig. 3. Comparison of Chaboche and Holloman stressstrain curves of single element test.

\subsection{Finite element analysis of wheel rim}

Today, finite element analysis has a wide usage area in mechanical engineering applications [10-14]. Cornering fatigue test is one of the fatigue tests, in which crack failures are investigated after definite cycles under dynamic loads [15]. In this study, finite element simulation of an aluminium alloy wheel was conducted. The finite element model has 95187 nodes and 373360 elements (Fig. 4). For this particular wheel the force applied on load arm was 3080 N. To simulate the rotation of the wheel around $y$-axis, force $F$ is separated into $x$ and $z$ components (Fig. 5).

$$
\begin{aligned}
& F_{x}=F \cos (2 \pi t), \\
& F_{z}=F \sin (2 \pi t) .
\end{aligned}
$$

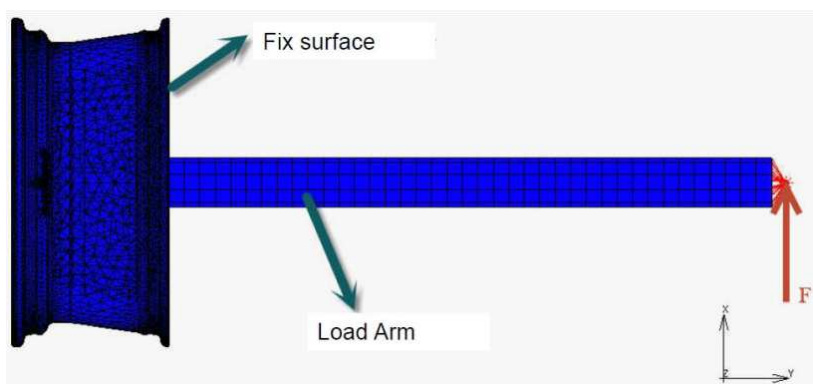

Fig. 4. Finite element model of wheel cornering test.

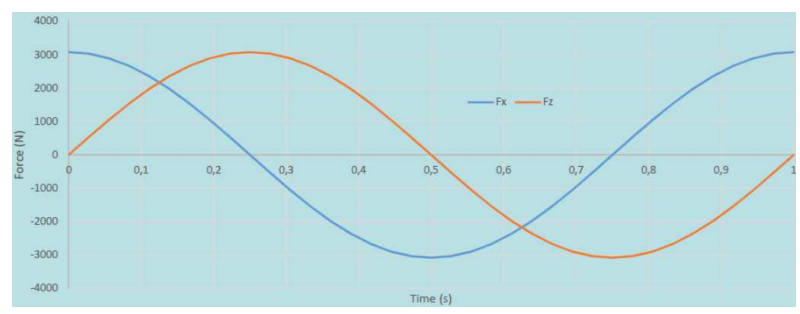

Fig. 5. Force-time curves of $F_{x}$ and $F_{z}$ components.

The results of finite element analysis show that the maximum equivalent Cauchy stress occurs in the 3rd loading step in node 10378 with a value of $99.86 \mathrm{MPa}$ (Fig. 6).

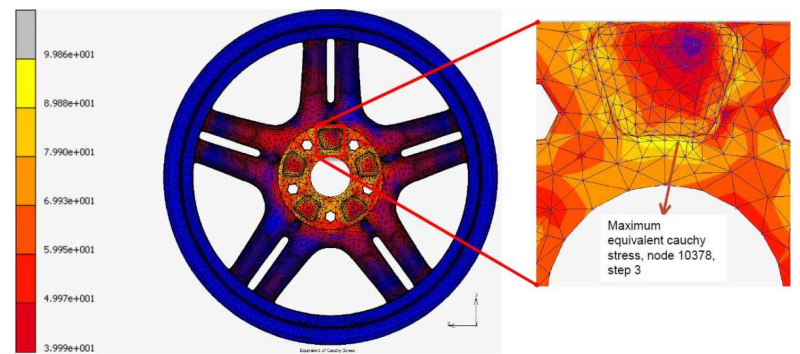

Fig. 6. Maximum equivalent Caucy stress location on the wheel.

\section{Conclusions}

In this study, it is aimed to obtain Chaboche kinematic hardening parameters using Holloman equation. Determination of the kinematic hardening parameters is a time consuming and a crucial process. The proposed methodology can calculate kinematic hardening parameters using 
Holloman equation, by curve-fitting. Obtained parameters are first verified with a single finite element test. Then, verified parameters are used in a wheel cornering test. Results show that the kinematic hardening parameters, obtained with proposed methodology, are effective and can be used in finite element analysis.

\section{References}

[1] Z.-G. Zheng, T. Sun, X.-Y. Xu, S.-Q. Pan, S. Yuan, Engin. Fail. Anal. 39, 124 (2014).

[2] G. Baffet, A. Charara, D. Lenchner, Control Engin. Pract. 17, 1255 (2009).

[3] D. Shang, X. Liu, Y. Shan, E. Jiang, Int. J. Fatigue 93, 173 (2016).

[4] X. Wang, X. Zhang, Int. J. Fatigue 32, 434 (2010).

[5] U. Kocabicak, M. Firat, Engin. Fail. Anal. 8, 339 (2001).
[6] F. Yoshida, T. Uemori, Int. J. Plasticity 18, 661 (2002).

[7] F. Barlat, J. Lian, Int. J. Plasticity 5, 51 (1989).

[8] J. Lemaitre, J.L. Chaboche, Mechanics of solid materials, Cambridge University Press, Cambridge 1990.

[9] J.H. Holloman, Trans. Am. Inst. Mining Metallalurgical Engin. 162, 268 (1945).

[10] O. Karacali, Acta Phys. Pol. A 130, 249 (2016).

[11] O. Karacali, Acta Phys. Pol. A 128, B-40 (2015).

[12] R.M. Nejad, Engin. Fail. Anal. 45, 449 (2014).

[13] J. Stearns, T.S. Srivatsan, A. Prakash, P.C. Lam, Mater. Sci. Engin. A 366, 262 (2004).

[14] M.M. Topac, S. Ercan, N.S. Kuralay, Engin. Fail. Anal. 20, 67 (2012).

[15] L. Shaohua, Y. Shaopu, C. Liqun, Appl. Math. Modell. 40, 6310 (2016). 